\title{
Missed Diagnosis of Subarachnoid Haemorrhage
}

\author{
Teiko Kawahigashi ${ }^{1}$, Taro Shimizu ${ }^{2}$, Takashi Kawabe ${ }^{1}$, Yoshitoshi Kida ${ }^{1}$, Kazunao Watanabe ${ }^{3}$ \\ ${ }^{1}$ Department of Emergency Medicine, Tokyo Nishi Tokushukai Hospital, Tokyo, Japan \\ ${ }^{2}$ Department of Diagnostic and Generalist Medicine, Dokkyo Medical University Hospital, Tochigi, Japan \\ ${ }^{3}$ Department of Surgery, Tokyo Nishi Tokushukai Hospital, Tokyo, Japan
}

Received: 06/05/2021

Accepted: 07/05/2021

Published: 31/05/2021

How to cite this article: Kawahigashi T, Shimizu T, Kawabe T, Kida Y, Watanabe K. Missed diagnosis of subarachnoid haemorrage. EJCRIM 2021;8: doi:10.12890/2021_002616.

Conflicts of Interests: The authors declare there are no competing interests.

Acknowledgements: We would like to thank Editage (www.editage.jp) for English language editing.

This article is licensed under a Commons Attribution Non-Commercial 4.0 License

\section{ABSTRACT}

A 79-year-old woman presented with left retro-orbital pain, headache and blurred vision. Based on negative radiological tests, lifethreatening conditions like subarachnoid haemorrhage (SAH) were ruled out and outpatient follow-up was planned. However, the patient returned to the hospital that night because of progressively declining consciousness and was diagnosed with SAH by head computed tomography. The diagnosis of SAH is often challenging, especially in cases with negative radiological results. We describe some strategies, other than radiological examination, for ruling out SAH, such as performing a lumbar puncture and repeating tests to take account of disease progression, and describe biases which can affect clinical decision-making.

\section{LEARNING POINTS}

- A high diagnostic error rate highlights the difficulty in diagnosing subarachnoid haemorrhage (SAH).

- Headache together with oculomotor nerve palsy is an important symptom of SAH.

- If SAH is suspected, further diagnostic measures including lumber puncture are warranted.

- A strategy of repeating tests to take account of disease progression would also be effective.

\section{KEYWORDS}

Emergency medicine, subarachnoid hemorrhage, diagnostic error

\section{CASE DESCRIPTION}

A 79-year-old woman presented with a 2-day history of left retro-orbital pain and headache, which had progressively worsened. The previous morning she had also experienced blurred vision and drooping of her left eyelid. The pain was associated with nausea without vomiting. The patient had a history of coronary artery bypass surgery, asthma and atrial fibrillation, which was treated with warfarin. Upon examination, she was alert and well oriented, with stable vital signs. Her visual field was intact and pupils were equally round and reactive to light, but she could not move her left eye laterally. Other neurological findings were normal.

To investigate the possibility of intracranial abnormalities such as subarachnoid haemorrhage (SAH), an unruptured intracranial aneurysm or pituitary apoplexy, the emergency physician performed non-contrast-enhanced computed tomography (CT) and magnetic resonance imaging (MRI) of the brain, which showed no evidence of haemorrhage or obvious aneurysms. The patient was referred to an ophthalmologist, who ruled out an acute attack of angle-closure glaucoma. The next morning, she visited a neurosurgeon and neurologist for further evaluation and was sent home without treatment because both physicians determined that she did not have emergent problems but continuous followup was required. 
However, she returned to the hospital that night with progressively declining consciousness. On arrival at hospital, she had a temperature of $36.5^{\circ} \mathrm{C}$, a Glasgow Coma Scale (GCS) score of 3/15 (E1V1M1), blood pressure of 180/70 mmHg, a heart rate of 65 beats per minute, and a respiratory rate of 18 breaths per minute with oxygen saturation $\left(\mathrm{SpO}_{2}\right)$ of $94 \%$ on room air. An emergent head CT revealed SAH (Fig. 1). She was intubated and transferred to another hospital for further treatment. The next day, magnetic resonance angiography revealed a $2 \mathrm{~mm}$ left middle cerebral artery bifurcation aneurysm. It was presumed that the aneurysm was the cause of the SAH.

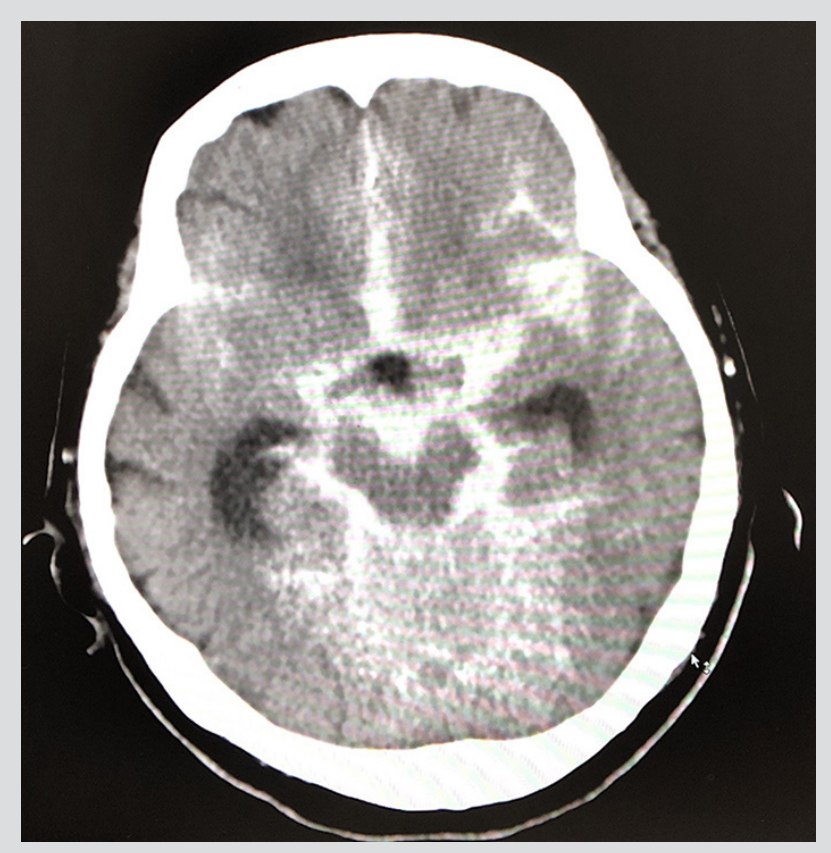

Figure 1. The second head CT image shows diffuse subarachnoid haemorrhage

\section{DISCUSSION}

$\mathrm{SAH}$ is a medical emergency. Diagnosis can be challenging because the initial presentation is variable and sometimes there are only limited and subtle signs. The rate of missed or delayed diagnoses ranges from $12 \%$ to $51 \%{ }^{[1,2]}$.

In this case, there were two possibilities regarding the onset of $\mathrm{SAH}$ : (a) the initial symptoms were caused by the unruptured intracranial aneurysm and the rupture of the aneurysm made symptoms worse; and (b) the aneurysm had ruptured at the time of the first visit but was missed. We consider the latter idea is more likely because a few patients with unruptured intracranial aneurysms experience acute headache $(6 \%)^{[3]}$. Laun et al. reported that palsy of one or several cranial nerves can occur in $9 \%$ of patients with SAH and that the oculomotor nerve was the most frequently affected ${ }^{[4]}$. Therefore, we should consider severe headache and oculomotor nerve palsy as important signs of SAH and perform other investigations such as lumbar puncture even if initial radiographic imaging does not show any evidence of haemorrhage. Alternatively, initial tests should be carefully repeated after a period of time to allow for disease progression, so called as "revisiting tests in time-axis perspective(RTA)"[5]. In this case, it may have been necessary to perform another CT or MRI, and follow-up with hospital admission, instead of sending the patient home.

In addition to the factors discussed above, clinician biases likely delayed the diagnosis, including rule bias and premature closure. Head CT has high sensitivity for detecting acute $\mathrm{SAH}$, especially when it is combined with MRI, but is not always accurate. However, we followed the results of imaging studies and paid little attention to the patient's symptoms. Furthermore, we did not perform further examinations even though the patient demonstrated severe symptoms suggesting SAH. Also, the patient presented to hospital as a walk-in case 2 days after symptom onset, which might have clouded the decision-making process. Kuchiki et al. reported that patients with SAH waited an average of 2.2 days before consulting a physician. They pointed out that a delay between symptom onset and presentation to hospital may mean physicians consider emergent disease like SAH less likely ${ }^{[3]}$.

Although there were some difficulties reaching the diagnosis, the patient's symptoms were associated with SAH based on her clinical course. As in this case, negative test results are common in daily practice, and so other diagnostic tests should be performed. 


\section{REFERENCES}

1. Oh SY, Lim YC, Shim YS, Song J, Park SK, Sim SY, et al. Initial misdiagnosis of aneurysmal subarachnoid hemorrhage: associating factors and its prognosis. Acta Neurochir (Wien) 2018;160(6):1105-1113.

2. Vermeulen MJ, Schull MJ. Missed diagnosis of subarachnoid hemorrhage in the emergency department. Stroke 2007;38(4):1216-1221.

3. Kuchiki H, Kokubo Y, Kondo R, Shinya S. Subarachnoid hemorrhage presenting mild headache. Surg Cereb Stroke (Jpn) 2014;42:122-126.

Laun A, Tonn JC. Cranial nerve lesions following subarachnoid hemorrhage and aneurysm of the circle of Willis. Neurosurg Rev 1988;11(2):137-141.

5. Kakimoto S, Sakamoto T, Hirosawa T, Shimizu T. How do we diagnose diseases with delayed imaging findings? Poster presentation at the 18th General Assembly of the Japanese Society of Hospital General Medicine, Okinawa, Japan, 2019 